\title{
How supervisor trust affects early residents' learning and patient care: A qualitative study
}

\author{
Brian C. Gin (D) - Stephanie Tsoi · Leslie Sheu · Karen E. Hauer (D)
}

Received: 5 November 2020 / Revised: 11 June 2021 / Accepted: 14 June 2021 / Published online: 23 July 2021

(C) The Author(s) 2021

\begin{abstract}
Introduction Trust between supervisors and trainees mediates trainee participation and learning. A resident (postgraduate) trainee's understanding of their supervisor's trust can affect their perceptions of their patient care responsibilities, opportunities for learning, and overall growth as physicians. While the supervisor perspective of trust has been well studied, less is known about how resident trainees recognize supervisor trust and how it affects them.

Methods In this qualitative study, 21 pediatric residents were interviewed at a single institution. Questions addressed their experiences during their first post-graduate year (PGY-1) on inpatient wards. Each interviewee was asked to describe three different patient care scenarios in which they perceived optimal, under-, and over-trust from their resident supervisor. Data were analyzed using thematic analysis.

Results Residents recognized and interpreted their supervisor's trust through four factors: supervisor, task, relationship, and context. Optimal trust was associated with supervision balancing supervisor availability and resident independence, tasks affording participation in decision-making, trusting relationships with
\end{abstract}

The authors Brian C. Gin and Stephanie Tsoi are joint first authors.

Supplementary Information The online version of this article (https://doi.org/10.1007/s40037-021-00674-9)

contains supplementary material, which is available to authorized users.

\section{B. C. Gin $(\bowtie) \cdot$. S. Tsoi}

Department of Pediatrics, University of California-San

Francisco, San Francisco, CA, USA

brian.gin@ucsf.edu

\section{Sheu · K. E. Hauer}

Department of Medicine, University of California-San

Francisco, San Francisco, CA, USA supervisors, and a workplace fostering appropriate autonomy and team inclusivity. The effects of supervisor trust on residents fell into three themes: learning experiences, attitudes and self-confidence, and identities and roles. Optimal trust supported learning via tailored guidance, confidence and lessened vulnerability, and a sense of patient ownership and team belonging.

Discussion Understanding how trainees recognize supervisor trust can enhance interventions for improving the dialogue of trust between supervisors and trainees. It is important for supervisors to be cognizant of their trainees' interpretations of trust because it affects how trainees understand their patient care roles, perceive autonomy, and approach learning.

Keywords Entrustment · Trust · Autonomy • Supervision $\cdot$ Trainee $\cdot$ Residency

\section{Introduction}

A growing body of literature identifies supervisor trust as a key component of trainees' experiential learning in the health professions. Trust can be considered as the affective and cognitive factors that influence a trustor's decision to delegate a responsibility to a trustee [1], whereas the term entrustment refers to a clinical supervisor's decision to transfer responsibility for a task to a trainee [2]. The trust a supervisor has in a trainee mediates opportunities for trainees to participate progressively and assume clinical independence [3]. Because effective trust requires both entrustment from the supervisor and engagement by the trainee, it is important to understand how trainees recognize and respond to their supervisors' trust.

Initial studies of entrustment focused on supervisors' perspectives [4-7], but more recently, the discus- 
sion around trust has shifted to trainees' perspectives [8-10]. For medical students, supervisor trust supports the scaffolding that generates opportunities for clinical learning and participation [11]. Studying resident trainees' perspectives of entrustment revealed actionable ways for trainees to earn trust from their supervising attending physician [12]. A study of how residents manage clinical uncertainty revealed a role for trusting relationships in promoting residents' autonomy and reciprocity of trust back to their supervisors [13]. This notion of mutual trust is thought to arise from early affective impressions, followed later by cognitive judgements from both supervisor and trainee viewpoints [14].

Less is known about how trainees recognize supervisor trust. Assessing trainees' understanding of their supervisors' trust is important for supervisors to know whether they are communicating trust effectively, and whether trainees are interpreting the intended degree of trust accurately. In addition, trainees' recognition and interpretation of supervisor trust may affect how they understand their roles in patient care, perceive autonomy, approach learning, and ultimately grow into independent physicians. How residents interpret and respond to supervisor trust is particularly important in the first year of postgraduate training (PGY-1) because trainees' early perspectives have the potential to affect their overall residency experience and development as physicians [15]. During this year, trainees gain increasing patient care responsibilities and, for the first time, are granted autonomy to act without direct supervision as physicians.

Several studies have corroborated five factors that physician supervisors consider when entrusting resident trainees: supervisor, trainee, relationship, task, and context [4-7]. While prior work has not directly applied these factors to the trainee perspective, similar themes can be seen in studies on trainee perspectives of feedback and autonomy [16, 17]. Utilizing these factors as a sensitizing framework [18], this study aimed to (1) understand how residents recognize supervisor trust and (2) explore the effects of residents' perceptions of supervisor trust on their learning and patient care. Clarifying the trainee perspective of trust during residency can improve how supervisors and trainees work together to deliver patient care and facilitate trainee learning and progression.

\section{Methods}

\section{Study design}

We conducted a qualitative interview-based study to investigate perceptions of pediatric residents during PGY-1.

\section{Participants and setting}

PGY-1 and 2 residents in the 3 -year pediatric residency at the University of California, San Francisco (UCSF) in the United States were eligible to participate after completing at least one month of inpatient pediatrics wards. During inpatient wards rotations, PGY-1 residents at UCSF are primarily supervised by "senior" residents (PGY-3). We invited all 60 PGY-1 and PGY-2 residents from the 2014-2015 academic year, and all 30 PGY-1 residents from the 2015-2016 academic year for individual interviews. Interviews occurred from February to October 2015 and took place during the first half of the academic year for PGY-1 participants, and after completion of the PGY-1 year for PGY-2 residents. The UCSF institutional review board deemed this study exempt (UCSF IRB File no. 14-15087).

\section{Interview guide and data collection}

Three authors (BG, LS, KEH) developed a semi-structured interview guide (see Appendix in the Electronic Supplementary Material [ESM]) based on the critical incident technique-a method chosen to identify salient experiences related to a broad range of trust [19]. We asked interviewees to recall patient care scenarios they experienced as PGY-1 residents on inpatient pediatrics wards. To capture a range of levels of trust, we asked them to recall scenarios with optimal trust, under-trust, and over-trust. Authors refined the interview guide for clarity through three pilot interviews, which were included in the final data set. A single author (BG) performed 21 interviews in person and by telephone, which were recorded, professionally transcribed, and de-identified. Interviews lasted on average $30 \mathrm{~min}$. We coded transcripts concurrently with data collection and stopped interviewing when we identified no further codes and scenario descriptions became multiply redundant, signaling sufficiency [20].

\section{Data analysis}

We performed thematic analysis using matrix exploratory methods designed to be generally applicable to all qualitative frameworks $[21,22]$. We chose thematic analysis to identify patterns related to our aims across different levels of trust [23]. First, BG created short summaries of each transcript and pulled direct quotes from each transcript and generated a preliminary descriptive code list. Three authors (BG, LS, KEH) read nine initial transcripts and revised these codes iteratively [24]. BG and ST applied these codes to the remaining transcripts and constructed a partially ordered meta-matrix [25] of coded excerpts grouped vertically by the level of trust (optimal/under/over). All authors participated in iterative discussion and data review to synthesize data into larger themes, focusing on trainee perceptions of supervisor behav- 
iors that supported trust, and on trainee perceptions of the effects of trust. We used Dedoose qualitative software for coding and analysis [26].

We considered the authors' reflexivity in this study, with particular acknowledgement of the medical hierarchy [27]. At the time of analysis one author (ST) was a UCSF pediatric resident. Another author (BG) was a senior resident (PGY-3) at study onset and subsequently a pediatric hospitalist fellow during data collection, with no direct responsibilities in evaluating trainees. Two authors (LS, KEH) are internal medicine attendings and educational scholars. Throughout data collection and analysis, we reflected on and discussed the impact of our roles, representing both trainee and supervisor viewpoints in adult and pediatric inpatient medicine. While we believe that our discussions were enriched by our representation of diverse stakeholder groups, we also strove to make inferences apart from these perspectives.

\section{Results}

Of 90 eligible residents, 32 volunteered to participate, and 21 were available for scheduled interviews. PGY-1 participants had been in residency training for an average of $3.5(S D=0.1)$ months at the time of their interview, and PGY-2 participants $19.6(S D=1.0)$ months. Participants included 17 (81\%) women, similar to $76 \%$ in the overall program.

We grouped our results according to our two research questions: how trainees recognize supervisor trust and how trainees are affected by their perceptions of trust. We identified four codes addressing how trainees recognized supervisor trust. These codes aligned with four of the five factors from our sensitizing framework (Hauer and colleagues' model of supervisor trust [3]) - supervisor, task, relationship, and context (see ESM, Table S1). Interviewees discussed the fifth factor of this model, the trainee, not in terms of their own contributions to trust, but rather by the effects of trust on themselves. Three additional codes described the effects of trust on PGY-1 trainees: learning experiences, attitudes and self-confidence, and identity and role (see ESM Tab. S2). Sorting interviewees' experiences by code and trust level revealed patterns described by the themes shown in both tables.

\section{How trainees recognize supervisor trust}

\section{Supervisor}

Trainees recognized different levels of trust based on the amount and type of support a supervisor provided when they faced acute or complex patients, unfamiliar cases, or difficult situations. Supervisors showed support through their preparation, teaching, availability, debriefing, and feedback. Trainees perceived optimal trust from their supervisors when they received what they perceived to be an appropriate amount of support. Trainees described feeling that their supervisors were invested in them when they spent time to thoroughly assess the trainee's skills and competence, and shared expertise relevant to the trainee's needs. Trainees recognized excessive supervisor support as under-trust. Trainees described behaviors such as underestimation of their capabilities by their supervisors, micromanaging, and redundancy in work. Trainees perceived over-trust when supervisors provided insufficient support, overestimated trainee capabilities, and were unavailable for teaching and guidance.

\section{Task}

Trainees recognized trust based on the type of task they were assigned to complete by their supervisors. The amount of trust perceived by the trainee was influenced by characteristics of the task, including complexity, acuity, sequencing of tasks over time, opportunity for autonomy, and associated risk to the patient. In optimal trust scenarios, trainees described being assigned tasks that matched their competence and appropriately promoted their growth. Trainees' roles and responsibilities were communicated clearly from the supervisor to the trainee and promoted critical thinking and participation in patient care. Trainees recognized under-trust when supervisors assigned tasks in ways that restricted their learning and growth, limiting their roles and responsibilities. This included exclusion from decision-making processes, and assignment of low acuity, quantity, and/or complexity of tasks based on the trainee's self-assessed competence. Trainees recognized over-trust when tasks exceeded their self-assessed competence. They described these tasks as either overly complex, involving a patient that was too acute, or necessitating a degree of responsibility that was above their expectations.

\section{Relationship}

Trainees recognized trust differently depending on the trainee-supervisor working relationship. Interpersonal dynamics, values, expectations, communication, and the amount of contact between trainee and supervisor affected the relationship that grounded trainee-supervisor trust. Optimal trust was fostered in relationships where the trainee and supervisor had mutual trust. Mutual trust was characterized by open and honest communication, the trainee feeling valued and receiving validation as a team member, shared expectations and mental models, mutual advocacy, and bidirectional feedback. Trainees felt optimally trusted when they had multiple opportunities to interact with their supervisors and form meaningful, longitudinal relationships with them. In situations of under-trust, trainees described relationships that felt hierarchical and even dictatorial. Trainees recalled feeling excluded from the team, experiencing oneway communication, or having unclear roles and expectations set between the trainee and the supervisor. 
Trainees recognized over-trust when working relationships were lacking or poor. These relationships were characterized by a lack of teamwork, poor or no communication, absence of shared values or expectations, and no trust in the supervisor.

\section{Context}

Trainees noted how the context of the team and the workplace influenced whether they felt optimally, under-, or over-trusted. Characteristics of the workplace included opportunities for either autonomy or dependence, workload, timing (i.e., day shift vs. night shift, during rounds or sign-out, rotation switch day), and workplace culture. Trainees recognized optimal trust when there was a supportive workplace with ample opportunities for trainee autonomy and growth, a collaborative environment with shared workload between trainees and supervisors, and a culture that encouraged learning and independent thinking. Effective collaborations considered the trainee's strengths while maintaining time and space for learning and autonomy. This differed from under-trust, where the context included barriers to participation and critical thinking, like lack of time and space for learning opportunities and autonomy, a culture of 'getting the work done' over learning, and timing early in the trainee-supervisor's block together when supervisors tended to supervise more directly. In overtrust, trainees described barriers to asking for or receiving help, and a workload that exceeded trainee capabilities either because of high census or inadequate staffing.

\section{How trust affects trainees}

Trainees described a range of effects stemming from their supervisors' trust in them. We summarized those effects into three main codes: learning experiences, attitudes and self-confidence, and identity and role, to identify the nine themes shown in ESM Tab. S2.

\section{Learning experiences}

Optimal trust created a favorable personal learning experience for trainees, as supervisors appropriately guided the trainee to acquire new skills and meet learning goals. Trainees felt motivated to learn when there was encouragement to be independent, autonomous, and ask for help. Optimal trust allowed trainees to grow as clinicians: "I felt that I learned more. I felt that I grew more ... my clinical suspicions were getting more validated ... Those interactions led to more opportunities to do more" (Participant no. [PN] 1). Under-trust made trainees feel restricted because of limited opportunities for independence or a feeling that learning was not prioritized. The trainee could not advance as a physician because of excessive supervision, redirection without guidance, and absence of constructive feedback. Over-trust created variable personal learning experiences for trainees, both positive and negative. Some felt compelled to compensate for missing teaching and feedback by self-teaching or asking other co-residents, nurses, or attendings for guidance, while others used the extra trust to work through challenges they may otherwise not have tried. "In terms of learning, there's pros and cons. ... The pro is that it really pushes you to decide something and act on it, ... but the con is that you feel like you're learning in the dark" (PN-9).

\section{Attitudes and self-confidence}

Optimal trust positively affected trainees' self-worth by promoting their self-confidence and allowing them to feel valued and empowered. With this level of trust, one trainee remarked "I can remember a lot of situations where the resident just said, 'I trust your clinical judgment. I think this is the right decision.' That gives you a little bit more confidence" (PN-9). Alternatively, supervisors who under-trusted their trainees left them feeling belittled, worthless, undermined, and with lower self-confidence. Over-trust had variable effects on trainees' attitudes and self-confidence. There were examples of over-trust that led to increased confidence if the trainee was able to complete the task successfully, but if the trainee was not able to navigate the scenario, this led to doubt, fear, vulnerability, and discomfort-"I just felt really uncomfortable because I felt like there were a lot of things that she expected me to be able to figure out on my own that I didn't... I always felt a little alone and vulnerable" (PN-19).

\section{Identity and role}

Optimal trust helped trainees establish their physician identity and have a defined role on the team. Trainees felt greater patient ownership and had more opportunities for participation in both the team as well as with direct patient care. Trainees described how their role in decision-making grew with optimal trust: "You know everyone else is thinking about the patient too, but I definitely felt like I was the one who was making the decisions" (PN-13). When trainees felt that their supervisors under-trusted them, they felt powerless, marginalized, and an unimportant part of the team. Their role felt minimized due to limited ownership over patient care and decision-making. Trainees who experienced over-trust felt they were given a role they did not deserve, often when their supervisor was busy. Trainees described feeling lost and worried about patient safety, but at times also willing to handle the increased autonomy and responsibility.

\section{Discussion}

This study brings new insights to trainee-supervisor trust by exploring how early resident trainees interpret and respond to different levels of their supervisors' trust. Our results show how four of the five factors from our sensitizing framework, based on the supervisor perspective [5], can be applied to describe 
trainees' recognition of supervisor trust. While our interview guide was based on these five factors, we intentionally used open-ended questions for the possibility of finding new factors; though we did not find new factors and the previously described categorization seemed to largely apply. Our findings illustrate how the supervisor and task factors directly influence perceived trust, while relationship and context contribute to the foundation for building trust. The balance of these factors creates a perceived level of trust that consequently affects the fifth factor, the trainee. We synthesize our results as a conceptual model in Fig. 1, which depicts how a trainee's recognition of trust is dependent on the appropriate balance between the amount of supervisor support and the acuity/complexity of a task within the given working relationship and context. The perceived trust level affects the trainee's learning experiences, attitudes and selfconfidence, and identity and roles established during their first year of residency. Altogether, trainees'
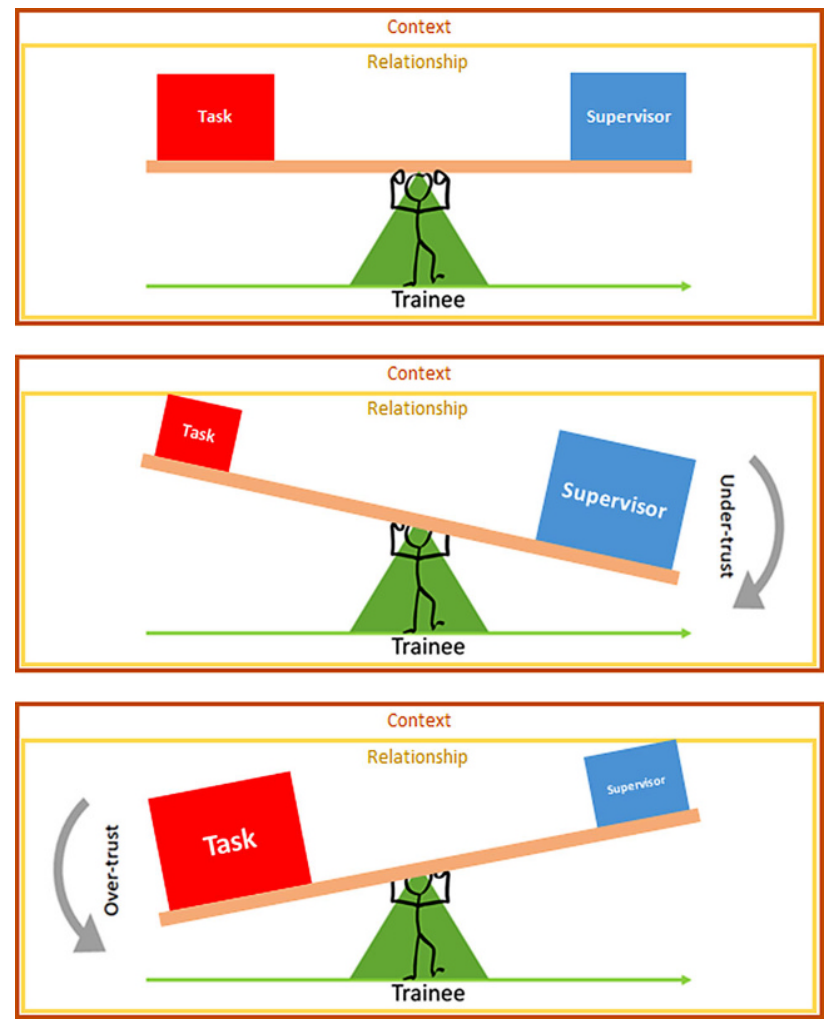

Fig. 1 Depiction of the interplay between the five factors of trust as perceived and received by the trainee. Trainees inferred different levels of trust based on the balance between task acuity and complexity and supervisor support. This balance could be shifted depending on the context and relationship factors. This balance impacts the trainee. a In optimal trust, trainees perceived appropriately balanced task acuity and complexity with supervisor support; as task acuity and complexity increased so did supervisor support and vice versa. b In under-trust, trainees recognized low task acuity and complexity with excessive supervisor support. c In overtrust, trainees received high task acuity and complexity with inadequate supervisor support responses to optimal supervisor trust seem to positively impact clinical learning and professional development.

Although the five factors were originally described to characterize supervisors' entrustment decisions about trainees $[5,28]$, the applicability of these factors to the trainee perspective is not surprising given their importance to the clinical learning environment $[29,30]$, the appearance of similar factors in models of bidirectional trust and social interaction [1, 13, 31], and the centrality of relationship formation in theories of supervision [32-35]. This alignment may allow for the development of a common dialect encompassing both directions of trust. Adopting these five factors as a language for trust may therefore facilitate development of shared mental models between supervisors and trainees to understand, recognize, and build trust.

Our proposed model of how a trainee recognizes supervisor trust (Fig. 1) could be used in curricular interventions designed to teach trust through the balance of these five factors. Recent discussions highlight the importance of the autonomy-supervision balance in clinical learning and its outcomes on how residents form professional identities, including their sense of patient ownership, decision-making ability, and confidence $[17,36]$. Sawatsky and colleagues showed that residents perceived supervisors to trust them when they were given autonomy, but also that too much autonomy could compromise learner and patient safety [17]. Our results suggest that trainees' understanding of their supervisor's trust comes from trainees' beliefs about how the five factors should interplay to achieve optimal trust. Therefore, learning interventions aimed at developing awareness of the five factors of trust could support appropriate promotion along the autonomy-supervision balance [7, 37-39]. This training might come in the form of early-residency curricula specifically addressing how to recognize and negotiate trust. Later, it could adapt to residents' roles as supervisors by discussing how to give recognizable trust, and how to address trainees' learning needs in the context of their drive for autonomy. Developing ways for supervisors and trainees to discuss trust openly may move discussions beyond trust's affective roots [14] - roots that may be particularly prone to unintended and unfair biases [40]. Involving trainees in explicit discussions about their entrustment may help to build trust grounded in objective decision variables based on trainee competencies rather than affective impressions [16].

Our findings highlighting the powerful impact trust can have on trainees' attitudes and self-confidence align with self-efficacy theory [41]. Self-efficacy in the setting of medical education is described as a taskspecific self-confidence that makes one feel more prepared, motivated, and capable [42]. Self-efficacy theory posits that trainees' perceptions of their own competence depend on certain key sources, like mas- 
tery experiences and physiological or affective states $[43,44]$. Interviewees positively referenced examples of these key sources when describing optimal trust, suggesting that optimal trust can increase trainees' self-efficacy. It remains to be determined whether trust or autonomy is the primary driver of self-efficacy in this context [17]. Additionally, since recent studies have suggested a relationship between selfefficacy and well-being, it would be interesting to study whether establishing optimal trust can improve well-being through higher self-efficacy [45].

This study has limitations. We examined a single program setting in a single specialty and conducted data collection five years ago; transferability of our results to other settings or specialties may be limited. Participants volunteered to be interviewed, possibly contributing a volunteer bias and/or more passionate feelings (either positive or negative) about the topic of trust. The majority of the participants were female, which is representative of pediatrics [46], but not all specialties, and may not accurately represent the valuation of trust, although there is no study to suggest a gender dependence of trust in medical education. Additionally, participants were interviewed at different times in their training (PGY-1 and PGY-2) and may have discussed scenarios with recall bias from subsequent experiences. Finally, representing trust as three categories (optimal, over-, and under-) may oversimplify its complexity, but our interviewees did appear to relate readily to this categorization, which enabled us to gather data on a range of experiences with trust.

Overall, this study provides a new perspective on supervisor trust from the early resident trainee viewpoint and explores the consequences of different levels of supervisor trust on trainees and their clinical growth. Adopting the five factors as a shared language between trainees and supervisors could help build trust and communicate it more effectively in the clinical learning environment. Open dialogue around finding an appropriate balance of supervisor support for a trainee's assigned task, developing trainee-supervisor relationships, and collaborating on contextual priorities may be ways to not only improve trust, but ultimately establish mutual trust. Trainees' perceptions of supervisor trust have important implications on their learning and patient care. Overall, our findings from the trainee perspective may help make trust more teachable, adaptable, and understandable for both trainees and their supervisors.

Acknowledgements We wish to thank Naike Bochatay, Mindy Ju, Sandrijn Van Schaik, Olle ten Cate, Christy Boscardin, and Patricia S. O'Sullivan for useful discussions. We thank Karen Sun, Darren Fiore, and Tim Kelly for support from the division of pediatric hospital medicine. Finally, we thank all the resident interviewees who made this work possible.

Conflict of interest B.C. Gin, S. Tsoi, L. Sheu and K.E. Hauer declare that they have no competing interests.
Open Access This article is licensed under a Creative Commons Attribution 4.0 International License, which permits use, sharing, adaptation, distribution and reproduction in any medium or format, as long as you give appropriate credit to the original author(s) and the source, provide a link to the Creative Commons licence, and indicate if changes were made. The images or other third party material in this article are included in the article's Creative Commons licence, unless indicated otherwise in a credit line to the material. If material is not included in the article's Creative Commons licence and your intended use is not permitted by statutory regulation or exceeds the permitted use, you will need to obtain permission directly from the copyright holder. To view a copy of this licence, visit http://creativecommons.org/licenses/by/4.0/.

\section{References}

1. Castelfranchi C, Falcone R. Trust theory: a socio-cognitive and computational model. Chichester: John Wiley \& Sons Ltd;2010.

2. ten Cate O. Trust, competence, and the supervisor's role in postgraduate training. BMJ. 2006;333:748-51.

3. Hauer KE, Ten Cate O, Boscardin C, Irby DM, Iobst W, O'Sullivan PS. Understanding trust as an essential element of trainee supervision and learning in the workplace. Adv Health Sci Educ Theory Pract. 2014;19:435-56.

4. Sterkenburg A, Barach P, Kalkman C, Gielen M, ten Cate O. When do supervising physicians decide to entrust residents with unsupervised tasks? Acad Med. 2010;85:1408-17.

5. Hauer KE, Oza SK, Kogan JR, et al. How clinical supervisors develop trust in their trainees: a qualitative study. Med Educ. 2015;49:783-95.

6. Sheu L, O'Sullivan PS, Aagaard EM, et al. How residents develop trust in interns: a multi-institutional mixed-methods study. Acad Med. 2016;91:1406-15.

7. ten Cate O, Hart D, Ankel F, et al. Entrustment decision making in clinical training. Acad Med. 2016;91:191-8.

8. Jason H. Celebrating 40 years of Medical Teacher: As the "last man standing" Ilook back to help us lookforward. Med Teach. 2018;40:331-6.

9. Abruzzo D, Sklar DP, McMahon GT. Improving trust between learners and teachers in medicine. Acad Med. 2019;94:147-50.

10. Sklar DP, McMahon GT. Trust between teachers and learners. JAMA. 2019;321:2157.

11. Karp NC, Hauer KE, Sheu L. Trusted to learn: a qualitative study of clerkship students' perspectives on trust in the clinical learning environment. J Gen Intern Med. 2019;34:662-8.

12. Pingree EW, Huth $\mathrm{K}$, Harper BD, et al. Encouraging entrustment: a qualitative study of resident behaviors that promote entrustment. Acad Med. 2020;95:1718-25.

13. Bochatay N, Bajwa NM. Learning to manage uncertainty: supervision, trust and autonomy in residency training. Sociol Health Illn. 2020;42:145-59.

14. Bonnie LHA, Visser MRM, Kramer AWM, van Dijk N. Insight in the development of the mutual trust relationship between trainers and trainees in a workplace-based postgraduate medical training programme: a focus group study among trainers and trainees of the Dutch general practice training programme. BMJOpen. 2020;10:e36593.

15. Bochatay N, Schaik S, O’Brien B. Medical trainees as job crafters: looking at identity formation through another lens. MedEduc. 2020;54:972-4.

16. Duijn CCMA, Welink LS, Mandoki M, ten Cate OT, Kremer WDJ, Bok HGJ. Am I ready for it? Students' perceptions 
of meaningful feedback on entrustable professional activities. Perspect Med Educ. 2017;6:256-64.

17. Sawatsky AP, Santivasi WL, Nordhues HC, et al. Autonomy and professional identity formation in residency training: a qualitative study. MedEduc. 2020;54:616-27.

18. Creswell JW. Research design: qualitative, quantitative, and mixed methods approaches. 4 th ed. Thousand Oaks: SAGE; 2013.

19. FitzGerald K, Seale NS, Kerins CA, McElvaney R. The critical incident technique: a useful tool for conducting qualitative research. JDentEduc. 2008;72:299-304.

20. Vasileiou K, BarnettJ, Thorpe S, Young T. Characterising and justifying sample size sufficiency in interview-based studies: systematic analysis of qualitative health research over a 15-year period. BMCMed Res Methodol. 2018;18:1-18.

21. Miles M, Huberman A, Saldaña J. Designing matrix and network displays. In: Qualitative data analysis: a methods sourcebook. Thousand Oaks: SAGE;2013. pp. 107-19.

22. Sawatsky AP, Ratelle JT, Beckman TJ. Qualitative research methods in medical education. Anesthesiology. 2019;131:14-22.

23. Kiger ME, Varpio L. Thematic analysis of qualitative data: AMEE guide No. 131. Med Teach. 2020;42:846-54.

24. Maxwell JA. Qualitative research design: an interactive approach. 3rd ed. Thousand Oaks: SAGE; 2013.

25. Miles MB, Huberman AM, Saldaña J. Methods of exploring. In: Qualitative data analysis: a methods sourcebook. Thousand Oaks: SAGE; 2013.pp. 121-59.

26. Dedoose. Dedoose, web application for managing, analyzing, and presenting qualitative and mixed method research data.. http:/ / www.dedoose.com. Accessed 17 May2021.

27. Reid AMM, Brown JM, Smith JM, Cope AC, Jamieson S. Ethical dilemmas and reflexivity in qualitative research. Perspect Med Educ. 2018;7:69-75.

28. Holzhausen Y, Maaz A, Cianciolo AT, ten Cate O, Peters H. Applying occupational and organizational psychology theory to entrustment decision-making about trainees in health care: a conceptual model. Perspect Med Educ. 2017;6:119-26.

29. Gruppen LD, Irby DM, Durning SJ, Maggio LA. Conceptualizinglearning environments in the health professions. Acad Med. 2019;94:969-74.

30. van Schaik SM, Reeves SA, Headrick LA. Exemplary learning environments for the health professions. Acad Med. 2019;94:975-82.

31. Kenny DA, La Voie L. The social relations model. Adv Exp Soc Psychol. 1984;18:141-82.

32. Graen GB, Uhl-Bien M. Relationship-based approach to leadership: development of leader-member exchange
(LMX) theory of leadership over 25 years-Applying a multi-level multi-domain perspective. Leadersh Q. 1995;6:219-47.

33. Werbel JD, Lopes Henriques P. Different views of trust and relational leadership: supervisor and subordinate perspectives. J Manag Psychol. 2009;24:780-96.

34. Knoll DL, Gill H. Antecedents of trust in supervisors, subordinates, and peers. J Manag Psychol. 2011;26:313-30.

35. Dulebohn JH, Bommer WH, Liden RC, Brouer RL, Ferris GR.A meta-analysis of antecedents and consequences of leader-member exchange: Integrating the past with an eye toward the future. J Manage. 2012;38:1715-59.

36. McCullough LB, Coverdale JH, Chervenak FA. Trustworthiness and professionalismin academic medicine. Acad Med. 2020;95:828-32.

37. Pugh D, Hatala R. Being a good supervisor: it's all about the relationship. Med Educ. 2016;50:395-7.

38. Gingerich A, Daniels V, Farrell L, Olsen SR, Kennedy T, Hatala R. Beyond hands-on and hands-off: supervisory approaches and entrustment on the inpatient ward. Med Educ. 2018;52:1028-40.

39. Core entrustable professional activities for entering residency.. https://www.aamc.org/system/files/c/2/484778epal3toolkit.pdf. Accessed 17 May 2021.

40. Teherani A, Hauer KE, Fernandez A, King TE, Lucey C. How small differences in assessed clinical performance amplify to large differences in grades and awards: a cascade with serious consequences for students underrepresented in medicine. Acad Med. 2018;93:1286-92.

41. Bandura A. Self-efficacy: Toward a unifying theory of behavioral change. Adv Behav Res Ther. 1978;1:139-61.

42. Klassen RM, Klassen JRL. Self-efficacy beliefs of medical students: a critical review. Perspect Med Educ. 2018;7:76-82.

43. Artino AR. Academic self-efficacy: from educational theory to instructional practice. Perspect Med Educ. 2012;1:76-85.

44. Cook DA, Castillo RM, Gas B, Artino AR. Measuring achievement goal motivation, mindsets and cognitive load: validation of three instruments' scores. Med Educ. 2017;51:1061-74.

45. Milam LA, Cohen GL, Mueller C, Salles A. The relationship between self-efficacy and well-being among surgical residents. J Surg Educ. 2019;76:321-8.

46. Active physicians by sex and specialty, 2019. https://www. aamc.org/data-reports/workforce/interactive-data/activephysicians-sex-and-specialty-2019. Accessed: 17 May 2021. 